Copyright (C) 2002 IEEE. Reprinted from [Engineering in Medicine and Biology, 2002. 24th Annual Conference and the Annual Fall Meeting of the Biomedical Engineering Society] EMBS/BMES

Conference, 2002. Proceedings of the Second Joint, vol.2, no., pp. 1185 vol.2, 2002

This material is posted here with permission of the IEEE. Internal or personal use of this material is permitted. However, permission to reprint/republish this material for advertising or promotional purposes or for creating new collective works for resale or redistribution must be obtained from the IEEE by writing to pubs-permissions@ieee.org. By choosing to view this document, you agree to all provisions of the copyright laws protecting it. 


\title{
IMAGE REGISTRATION FOR INTERVENTIONAL MRI-GUIDED MINIMALLY INVASIVE TREATMENT OF PROSTATE CANCER
}

\author{
Baowei Fei ${ }^{1}$, Daniel T. Boll ${ }^{2}$, Jeffery L Duerk ${ }^{1,2}$, David L Wilson ${ }^{1,2}$ \\ ${ }^{1}$ Department of Biomedical Engineering, Case Western Reserve University, Cleveland, OH, USA \\ ${ }^{2}$ Department of Radiology, University Hospitals of Cleveland, Cleveland, OH, USA
}

\begin{abstract}
We are investigating automatic image registration methods that can be used for interventional magnetic resonance imaging (iMRI) guided radiofrequency (RF) thermal ablation of prostate cancer. We tested the ability of slice-to-volume registration between iMRI slice images and high-resolution MRI volumes. Images were acquired from a conventional $1.5 \mathrm{~T}$ and an interventional $0.2 \mathrm{~T}$ MRI system. We evaluated the registration quality by calculating 3D displacement on a voxelby-voxel basis over a volume of interest between slice-to-volume registration and volume-to-volume registration that was previously shown to be quite accurate. Visual inspections such as color overlay and contour overlap were also used for registration evaluation. More than 300 registration experiments were performed on MR images of volunteers. Results showed that the registration was quite robust and accurate $(<2 \mathrm{~mm})$ for the transverse images covering the prostate.

Keywords: medical imaging, image registration, interventional magnetic resonance imaging (iMRI), prostate cancer.
\end{abstract}

\section{INTRODUCTION}

We currently use interventional MRI as guidance for RF thermal ablation of abdominal cancer. In the case of the prostate, the tumor is not reliably identified with MRI. Other functional imaging techniques are required. Potential methods include SPECT imaging and MR spectroscopy. To incorporate the functional images with interventional MRI tumor targeting, one can first register the low-resolution functional images with high-resolution MR volumes. Then by registering high-resolution MR volume with nearly realtime iMRI acquisitions, we can map both the functional data and high-resolution anatomic information to interventional images for improved tumor targeting. From our previous success with MR volume-to-volume (VV) registration [1] and slice-to-volume (SV) registration using simulated iMRI images [2], we are further testing the SV registration ability using real iMRI images in this study.

\section{MATERIALS AND METHODS}

We acquired high-resolution MR volumes from three volunteers using a $3 \mathrm{D}$ rapid gradient echo sequence (PSIF) with 9.4/5.0/60 (TR/TE/FA) and a conventional 1.5 T MR system (Siemens Magnetom Symphony,Erlangen, Germany). Volunteers laid supine similar to the diagnostic position in routine MR scanning. Two volumes were acquired for each volunteer. Volume images were normally $256 \times 256 \times 128$ with the voxel size of $1.4 \times 1.4 \times 1.5-\mathrm{mm}$.

We also acquired images from the same volunteers using a clinical 0.2 T C-arm interventional MR system (Siemens Open Symphony, Erlangen, Germany). Volunteers were supine with the legs supported similar to the lithotomy position used in prostate therapies. This position should provide access for needle insertion in brachytherapy or RF thermal ablation. We used a 3D PSIF with 25/13/60 (TR/TE/FA) for volume acquisitions and 2D PSIF with
15.2/7.4/45 (TR/TE/FA) for slice acquisitions. Slice images were $128 \times 128$ with pixel size of $2.8 \times 2.8-\mathrm{mm}$ and with effective slice thickness of $5 \mathrm{~mm}$. For each volunteer, we acquired 2 volumes and 25 slices covering the prostate. They included 15 transverse, 5 coronal, and 5 sagittal slices. We called these images as iMRI volume or real iMRI slices. We call them real iMRI slices to differentiate from previous experiments on simulated iMRI slices.

We performed registration experiments using an automatic algorithm with special features important for robustness for MR pelvic images [1]. First, we performed VV registration between high-resolution MRI and iMRI volumes. Second, we extracted slices from an iMRI volume and registered the slices with the high-resolution MRI volume. We evaluated SV registrations by calculating $3 \mathrm{D}$ displacement on a voxelby-voxel basis over a volume of interest (VOI) between SV and VV registration [2]. Third, we tested the SV registration between real iMRI slices and high-resolution MRI volumes. We used visual inspections such as color overlay and contour overlap to evaluate the SV registration quality.

\section{RESULTS}

We performed over 300 registration experiments. The VV registration of MRI and iMRI volumes is quite accurate with prostate centroid typically less than $1 \mathrm{~mm}$. As compared to the VV results, the VOI displacement of SV registration is less than $2 \mathrm{~mm}$ for all transverse slices covering the prostate. Most coronal slices (86\%) gave an accuracy of less than $2.5 \mathrm{~mm}$. Nearly half of sagittal slices $(46 \%)$ gave an accuracy of less than $5 \mathrm{~mm}$. This suggested that transverse slices were the best. Inspection indicates that bladder and/or rectal filling interfered with registration of coronal and sagittal slices because of deformation. Visual inspection of SV registration between iMRI slices and high-resolution MRI volumes demonstrated similar results.

\section{CONCLUSION}

It is quite feasible to use SV image registration to aid the application of interventional MRI-guided RF thermal ablation of prostate cancer. We are beginning to explore these applications in dog experiments.

\section{ACKNOWLEDGMENT}

The algorithm developed in this research was supported by NIH grants R01-CA84433-01 to DLW, R33-CA88144-01 to JLD, and DOD Award DAMD17-02-1-0230 to B. Fei.

\section{REFERENCES}

[1] B. Fei, A. Wheaton, Z. Lee, J.L. Duerk, D.L. Wilson. "Automatic MR volume registration and its evaluation for the pelvis and prostate." Physics in Medicine and Biology, 47: 823-838, 2002.

[2] B. Fei, A. Wheaton, Z. Lee, K. Nagano, J.L. Duerk, D.L. Wilson.

"Robust registration algorithm for interventional MRI guidance for thermal ablation of prostate cancer." Proceedings of SPIE Medical Imaging on

Visualization, Display, and Image-Guided Procedures, 4319: 53-60, 2001. 